\title{
A desnutrição e obesidade no Brasil: o enfrentamento com base na agenda única da nutrição
}

\author{
Malnutrition and obesity in Brazil: \\ dealing with the problem through \\ a unified nutritional agenda
}

\footnotetext{
${ }_{1}$ Coordenação-Geral da Política de Alimentação e Nutrição, Ministério da Saúde, Brasília, Brasil. 2 Observatório de Políticas de Segurança Alimentar e Nutrição, Brasília, Brasil.

Correspondência J. G. Coutinho Coordenação Geral da Política de Alimentação e Nutrição, Departamento de Atenção Básica, Secretaria de Atenção à Saúde, Ministério da Saúde.

SEPN 511, bloco C, Edifício Bittar IV, $4^{\circ}$ andar, Brasília, DF 70750-543, Brasil. janinegc@yahoo.com janine.coutinho@saude.gov.br
}

\section{Abstract}

Brazil, like other developing countries, is currently experiencing a nutritional transition that is often determined by poor eating. Population-based surveys have shown a continuing downward trend in undernutrition in this country, together with increases in excess weight at different stages of life. Monitoring the nutritional profile helps generate an attitude of surveillance and orientation of health promotion activities. The Family Health Strategy, together with various situational aspects in Brazil, has shown tangible results in the reduction of undernutrition; still, poor eating has played a major role in the increased prevalence of obesity and other chronic non-communicable diseases, besides having a heavy impact on individual quality of life and placing a significant burden on the Unified National Health System. Intervening in this contemporary public health dilemma requires prioritizing a unified nutritional agenda focused on the entire course of life, breaking the vicious circle that begins during the intrauterine period and lasts throughout life.

Nutritional Epidemiology; Nutritional Transition; Chronic Diseases
Janine Giuberti Coutinho 1,2

Patrícia Chaves Gentil 1

Natacha Toral 1

\section{Introdução}

A presença da desnutrição, deficiência de micronutrientes, excesso de peso e outras doenças crônicas não transmissíveis coexistindo nas mesmas comunidades e, muitas vezes no mesmo domicílio, caracteriza a transição nutricional 1 . Esse fenômeno é traduzido em um dos maiores desafios para as políticas públicas no momento e exige um modelo de atenção à saúde pautado na integralidade do indivíduo com uma abordagem centrada na promoção da saúde.

Diversos estudos têm demonstrado que o Brasil, assim como outros países em desenvolvimento, convive com a transição nutricional, determinada freqüentemente pela má-alimentação 2,3 . Ao mesmo tempo em que se assiste à redução contínua dos casos de desnutrição, são observadas prevalências crescentes de excesso de peso, contribuindo com o aumento das doenças crônicas não transmissíveis. A essas são associadas as causas de morte mais comuns atualmente. Segundo a Organização Mundial da Saúde (OMS), a hipertensão arterial e a obesidade correspondem aos dois principais fatores de risco responsáveis pela maioria das mortes e doenças no mundo 4 . No Brasil, as doenças cardiovasculares correspondem à primeira causa de morte há pelo menos quatro décadas, acompanhada de um aumento expressivo da mortalidade por diabetes e ascensão de algumas neoplasias malignas 5 . 
As doenças crônicas não transmissíveis, por serem de longa duração, são as que mais demandam ações, procedimentos e serviços de saúde, gerando no Brasil uma sobrecarga do Sistema Único de Saúde (SUS). Estima-se que os gastos do Ministério da Saúde com atendimentos ambulatoriais e internações em função das doenças crônicas não transmissíveis sejam de aproximadamente $\mathrm{R} \$ 7,5$ bilhões por ano ${ }^{6}$. A obesidade, a hipertensão e o diabetes são propiciados pelo perfil alimentar encontrado entre as famílias brasileiras, em que há uma participação crescente de gorduras em geral, gorduras de origem animal e alimentos industrializados ricos em açúcar e sódio e a diminuição de cereais, leguminosas, frutas, verduras e legumes 7 .

Os dados de tendência de consumo são favoráveis à reversão dos problemas associados à desnutrição, com o aumento da disponibilidade de calorias per capita e o aumento da participação de alimentos de origem animal na alimentação 7. Por outro lado, ainda persistem no Brasil as formas mais severas da desnutrição, especialmente o déficit de estatura por idade. Este é mais grave nas regiões Norte e Nordeste, mas também presente em bolsões de pobreza nas demais regiões, o que caracteriza a desnutrição, sem dúvida, como um fruto da desigualdade social e pobreza do país.

Para atuar neste dilema da saúde pública contemporânea, é necessário priorizar uma agenda única de nutrição, no enfoque do curso da vida, quebrando um ciclo vicioso que se inicia ainda no período intra-uterino, e se perpetua ao longo da vida.

\section{Perfil nutricional da população brasileira}

Os resultados de inquéritos populacionais realizados no Brasil desde a década de 70 têm apresentado uma redução das prevalências de baixo peso em ambos os sexos, em diferentes fases da vida e em todas as regiões do país. Segundo o $E s$ tudo Nacional da Despesa Familiar (ENDEF), realizado em 1974-1975, 7,2\% dos homens e 10,2\% das mulheres apresentavam déficits ponderais ? Já na Pesquisa de Orçamentos Familiares (POF) de 2002-2003 verificou-se uma prevalência de baixo peso de $2,8 \%$ e $5,4 \%$ entre homens e mulheres, respectivamente 7 . Segundo a OMS, prevalências de baixo peso de até $5 \%$ são consideradas aceitáveis em países em desenvolvimento ${ }^{8}$. Nesse sentido, afirma-se que o déficit ponderal em adultos não é mais considerado um problema de saúde pública no Brasil.

Entre adolescentes, os resultados de inquéritos têm mostrado freqüências relativamente baixas de déficits ponderais, alcançando 3,7\% de acordo com a última POF. Nesse grupo etário, destaca-se particularmente a redução intensa e contínua do déficit de estatura nas últimas décadas. Os dados do ENDEF e da última POF apresentam um declínio da prevalência de déficit de altura de $33,5 \%$ para $10,8 \%$ entre os meninos, e de $26,3 \%$ para $7,9 \%$ entre as meninas 9 .

No caso das crianças menores de cinco anos, o déficit de peso por idade foi reduzido nacionalmente de 16,6\%, segundo o ENDEF, para 4,6\%, de acordo com os dados da POF de 2002-2003. Nas regiões Norte (área urbana) e Nordeste, a prevalência de déficit de peso partia de valores mais altos, de $21,7 \%$ a $24,9 \%$ no ENDEF, e foi reduzida de forma contínua ao longo dos inquéritos, alcançando $6,7 \%$ e $5,4 \% 9$.

Diversos fatores têm contribuído para a redução do déficit ponderal entre as crianças brasileiras nos últimos anos. Além dos ganhos econômicos, houve uma grande expansão dos serviços públicos de saneamento e programas de saúde, gerando uma ampliação da cobertura da assistência à saúde na população brasileira. Nesse contexto, cabe ressaltar a organização da atenção básica no SUS por meio da Estratégia Saúde da Família, em franca expansão desde 199410.

A Estratégia Saúde da Família tem como premissa o atendimento dos indivíduos e famílias de forma integral e contínua, desenvolvendo ações de promoção e proteção e recuperação da saúde e tem como objetivo reorganizar a prática assistencial, centrada no hospital, passando a enfocar a família em seu ambiente físico e social. Corresponde a um modelo de atenção que pressupõe o reconhecimento de saúde como um direito expresso na melhoria das condições de vida. No tocante à área da saúde, essa melhoria é traduzida em serviços mais resolutivos, integrais e, sobretudo, humanizados. Um importante impacto da Estratégia Saúde da Família é observado sobre a saúde das crianças: um aumento de 10\% na cobertura da Estratégia Saúde da Família resulta numa redução de $4,6 \%$ na taxa de mortalidade infantil 11.

De Onis et al. 12 apontam que o melhor indicador global do bem-estar da criança é o crescimento e, ainda, que o acompanhamento do crescimento não é adotado somente para a avaliação da saúde e do estado nutricional de uma criança, mas também corresponde a um excelente indicador de desigualdade nas populações. Podese inferir que a desnutrição é um dos produtos da desigualdade social que ainda prevalece no Brasil. Para superar essa situação perversa que compõe o panorama brasileiro da má-nutrição, são exigidas medidas eficientes e urgentes para o 
combate à pobreza e à fome por meio da implementação de políticas de inclusão social.

O Brasil e outros 189 países são signatários dos Objetivos do Desenvolvimento do Milênio que tem como referencial o alcance de um processo de desenvolvimento inclusivo e justo como prioridade para a eliminação da pobreza. Foram determinados oito objetivos, 18 metas e 48 indicadores. No Brasil essas metas foram redimensionadas a fim de acelerar os esforços do governo e da sociedade na reversão do quadro da pobreza e fome. É também importante ressaltar que as metas brasileiras são mais ambiciosas do que as metas propostas pela Organização das Nações Unidas (ONU). O primeiro objetivo é erradicar a pobreza e a fome.

Os desafios a serem enfrentados resultam, destacadamente, da falta de acesso aos alimentos, decorrentes do baixo poder aquisitivo de milhões de brasileiros. O problema de acesso é agravado por uma série de outros fatores, como condições inadequadas de saneamento básico, baixos níveis de educação e serviços de saúde deficientes. A fome e a desnutrição no país não se devem à escassez de produção de alimentos. Ao contrário, a agricultura nacional produz mais do que o suficiente para suprir as necessidades da população brasileira. Segundo dados da Organização das Nações Unidas para Agricultura e Alimentação (FAO), a disponibilidade de alimentos no Brasil aumentou sucessivamente e se situa no patamar de cerca de 3 mil quilocalorias (kcal) por pessoa dia 13 .

O Brasil é um país heterogêneo em relação à distribuição dos determinantes sócio-econômicos da desnutrição. A distribuição regional da pobreza mostra variações importantes, destacando-se a freqüência duas a três vezes maior de pobres, nas regiões Norte e Nordeste do que nas regiões Sudeste, Sul e Centro-Oeste 14. Contraste bem evidente ocorre, por exemplo, entre a população urbana do Sudeste, em que apenas um em cada sete indivíduos é pobre, e a população rural do Nordeste, em que três em cada cinco indivíduos são pobres. A distribuição regional da desnutrição segue a mesma distribuição da pobreza, determinando, ainda com maior intensidade, as desvantagens das regiões Norte e Nordeste. Pesquisa realizada em 1996 mostrou freqüência duas a três vezes maior de crianças com baixa estatura no Norte e Nordeste do que nas regiões do Centro-Sul do país 15.

Um dos mais relevantes focos de medidas deve ser a prevenção do déficit estatural na população infantil, considerado como produto da fome crônica, da desigualdade social e da miséria brasileiras 16. Este corresponde a uma das características mais significativas da desnutrição 17 e representa o efeito cumulativo do estresse nutricional sobre o crescimento esquelético. Continua a ser um dos problemas importantes de saúde pública, em razão das conseqüências desastrosas para o crescimento, desenvolvimento e até para a sobrevivência das crianças. A baixa estatura tem sido relacionada ao atraso na capacidade intelectual, baixo rendimento escolar, menor capacidade física para o trabalho, além de ser considerado como um determinante do maior risco para gerar crianças com baixo peso ao nascer entre as mulheres.

Dentre as estratégias possíveis para o mapeamento da desnutrição no país é a realização de “Chamadas Nutricionais". Elas são estratégias vinculadas às Campanhas de Imunização de Poliomielite (Programa Nacional de Imunizações, da Secretaria de Vigilância em Saúde), que permitem uma mobilização para fins de atitude de vigilância dos estados e principalmente dos municípios sobre a importância do acompanhamento do crescimento infantil. Além disso, permite a obtenção de informações sobre indicadores antropométricos e indicadores de consumo em crianças menores de cinco anos.

Em 2005, foi realizada a "Chamada Nutricional” na região do semi-árido brasileiro pelo Ministério do Desenvolvimento Social e Combate à Fome, em parceria com o Ministério da Saúde. No evento observou-se que $6,6 \%$ das crianças apresentavam déficit estatural (com valores abaixo do desvio-padrão 2 da mediana da população de referência). Diversas variáveis sócio-econômicas foram relacionadas à baixa estatura, sendo as maiores prevalências observadas entre crianças de um ano de idade (11\%), de famílias nas quais eram realizadas menos de três refeições ao dia (16,2\%) e que tinham mães sem instrução $(14,8 \%) 18$.

No entanto, uma avaliação feita em diversos países por meio de um banco de dados sobre crescimento infantil da OMS indica que, apesar de o Brasil apresentar déficits estaturais importantes, eles ficam abaixo da média global $(24,1 \%)$ e da média das nações em desenvolvimento $(26,5 \%)$. Neste estudo o Brasil registrou resultados melhores que a maioria dos países sul-americanos estudados (Bolívia, Colômbia, Guiana, Peru, Venezuela) - fica em posição desfavorável somente em relação ao Chile e ao Uruguai 12 .

O outro lado da transição nutricional da população brasileira refere-se às prevalências crescentes do excesso de peso e de obesidade. Os inquéritos populacionais têm mostrado um aumento expressivo do problema entre os homens, em todas as regiões brasileiras, alcançando $41 \%$ de excesso de peso e $8,8 \%$ de obesidade. Entre as mulheres, após um aumento de cerca de $50 \%$ en- 
tre a década de 70 e final dos anos 80 , observa-se certa estabilidade nas prevalências, mas 39,2\% e $12,7 \%$ apresentavam excesso de peso e obesidade, respectivamente, em 2002-2003 7 .

\section{Agenda única da nutrição}

O dilema atual da nutrição em saúde pública é lidar ao mesmo tempo com situações aparentemente contraditórias, como a desnutrição e a obesidade e suas implicações. Organismos internacionais vêm referenciando que para o enfrentamento da transição nutricional é necessário colocar na pauta uma série de políticas articuladas numa "agenda única de nutrição", mediante a promoção da alimentação saudável, no enfoque do curso da vida 1 .

Isso porque a promoção da alimentação saudável constitui-se numa das estratégias de saúde pública de vital importância para o enfrentamento dos problemas alimentares e nutricionais do contexto atual. Deve consistir em uma abordagem integral capaz de prevenir ao mesmo tempo as doenças causadas por deficiências nutricionais - reforçando a resistência orgânica para as doenças infecciosas - e em uma redução da prevalência do excesso de peso e das outras doenças crônicas não transmissíveis associadas 19 .

O enfoque do curso da vida foi desenvolvido nas últimas décadas, com base em estudos de coortes em diversos países, inclusive no Brasil, sugerindo que exposições nutricionais, ambientais e padrões de crescimento durante a vida intra-uterina e nos primeiros anos de vida podem ter efeitos importantes sobre as condições de saúde do adulto. $\mathrm{O}$ retardo de crescimento intra-uterino e o ganho de peso excessivo nos primeiros anos de vida têm sido associados em muitas pesquisas com o risco aumentado para obesidade, hipertensão, síndrome metabólica, resistência insulínica e morbimortalidade cardiovascular na vida adulta, entre outros desfechos desfavoráveis 20. Esse ciclo perverso está ilustrado na adaptação do modelo proposto por Darnton-Hill et al. 21 , conforme Figura 1.

Considerando que o início desse ciclo perverso está na má-nutrição intra-uterina e na desnutrição infantil, as estratégias clássicas de saúde pública, como o pré-natal para as gestantes, acompanhamento do crescimento e desenvolvimento, fomento do aleitamento materno exclusivo até o sexto mês, complementar até o segundo ano de vida, e ainda uma alimentação complementar adequada, devem ser priorizadas e fortalecidas em âmbito local de atenção à saúde. Ou seja, ampliar e, sobretudo, qualificar o acesso aos serviços de saúde e a cobertura da atenção à saúde, durante o período gestacional e a infância deveria ser a garantia de melhor estado nutricional e a possibilidade de eliminar a desnutrição em áreas e grupos populacionais específicos.

No Brasil, a Política Nacional de Alimentação e Nutrição (PNAN), criada em 1999, explicitou o papel do setor saúde para a agenda única de nutrição, considerando como pressupostos o direito humano à alimentação adequada e à segurança alimentar e nutricional 22. Dentre as sete diretrizes propostas, três delas merecem destaque: (1) o monitoramento da situação alimentar e nutricional da população brasileira; (2) a prevenção e controle das carências nutricionais e das doenças associadas à alimentação e à nutrição e (3) a promoção de práticas alimentares e estilos de vida saudáveis.

A criação da PNAN pautou-se na necessidade de recolocar a nutrição no cenário político, estruturar as ações de alimentação e nutrição no setor saúde e fomentar uma rede de apoio institucional nos estados e municípios brasileiros e, ainda, com a sociedade civil organizada e a academia. Além disso, veio fortalecer o setor numa perspectiva intersetorial, apontando sua interface direta com o Sistema Nacional de Segurança Alimentar e Nutricional (SISAN), discutido desde 2006, com a publicação da Lei Orgânica de Segurança Alimentar e Nutricional (LOSAN), que se fundamenta mediante a articulação de setores governamentais, a sociedade civil, a academia e o setor produtivo 22,23 .

As Tabelas 1 e 2 apontam exemplos de ações/ estratégias que devem ser priorizadas ou fortalecidas pelo setor saúde, nas três esferas de governo, na concepção de uma agenda única de nutrição.

Dentre as ações elencadas merecem destaque as medidas de promoção da alimentação saudável. Organizar essas ações é um dos desafios que se impõem atualmente. Isso porque promover uma alimentação saudável implica desenvolver mecanismos que apóiem os sujeitos a adotar modos de vida saudáveis, revendo hábitos alimentares considerados pouco saudáveis, num contexto em que a globalização e a urbanização caracterizam-se como movimentos incessantes e contínuos, altamente dificultadores do estabelecimento de práticas alimentares saudáveis, e que impactam interesses econômicos contrários à saúde pública.

Assim, é fundamental associar medidas de incentivo voltadas ao indivíduo como as estratégias de informação, campanhas e educação alimentar e nutrição; com medidas de apoio que auxiliem os indivíduos a fazerem suas escolhas alimentares mais saudáveis, como por exemplo: 


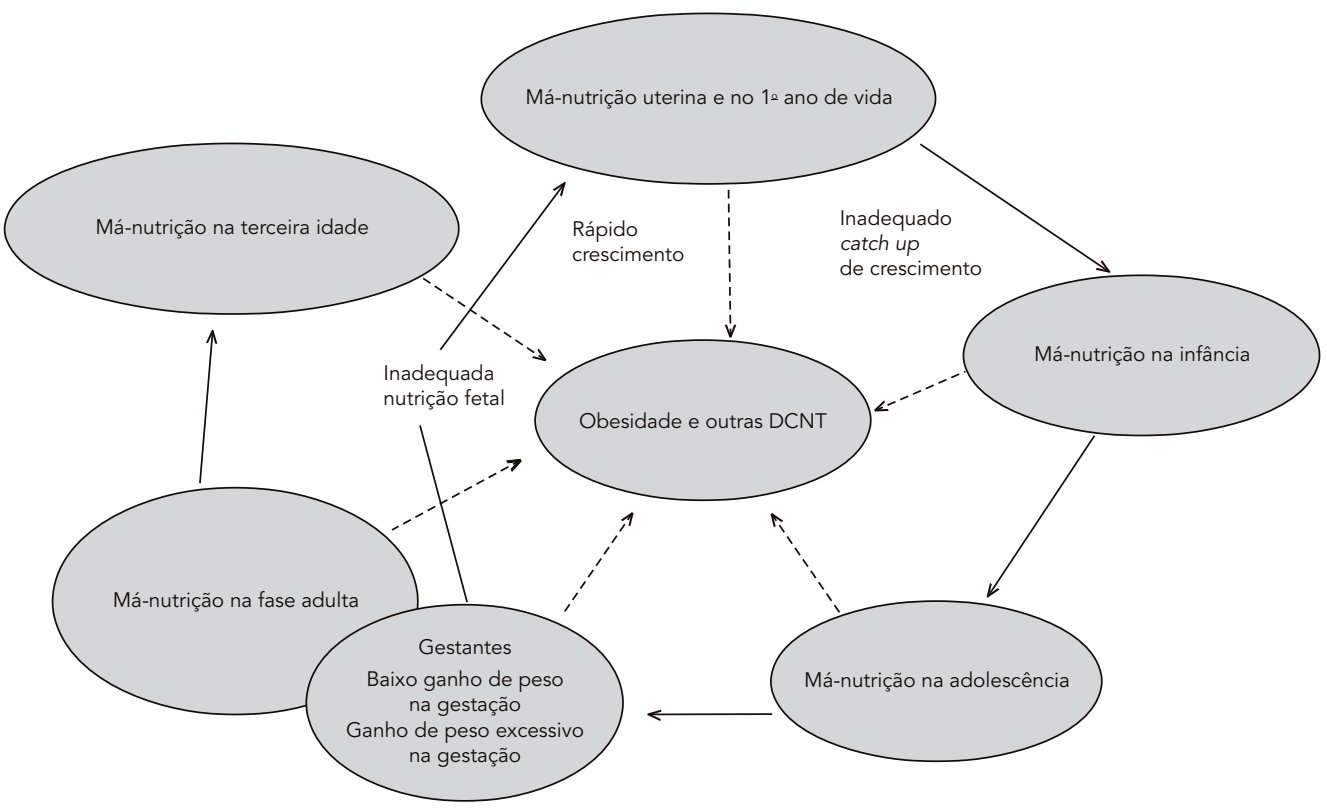

Adaptado de Darnton-Hill et al. 21.

DCNT: doenças crônicas não transmissiveis.

rotulagem nutricional dos alimentos, incentivos voltados à produção dos alimentos, comercialização, abastecimento e o acesso físico a alimentos mais saudáveis 19,24,25.

As medidas de proteção também assumem lugar essencial no contexto da promoção da alimentação saudável, pois implicam medidas regulatórias que protegem a população de práticas abusivas, como por exemplo: as práticas de marketing e publicidade de alimentos ricos em açúcar, gorduras e sal, focadas no público infantil, que interferem sobremaneira nos hábitos alimentares das crianças; no comércio e oferta desses alimentos no ambiente escolar, que deve ser um ambiente de formação de hábitos mais saudáveis; e na comercialização de alimentos processados, que devem ter seus teores de açúcar, gorduras e sal limitados, em virtude da má qualidade nutricional dos que estão sendo comercializados atualmente 19,24,25.

Tal discussão foi difundida mundialmente e tornada oportuna a partir da Estratégia Global de Promoção da Alimentação Saudável, Saúde e Atividade Física, capitaneada pela OMS e discutida na Assembléia Mundial, em 2005. O Brasil foi um dos países que se tornaram signatários, favorecendo a inserção na pauta política, setorial e intersetorial da discussão da obesidade no contexto da transição nutricional, bem como as medidas proposta na agenda única da nutrição 24 .

Em nível local, a concretização das ações de alimentação e nutrição em uma agenda única pode ser potencializada na atenção primária à saúde. Esse é um espaço privilegiado para o desenvolvimento das ações de incentivo e apoio à adoção de hábitos alimentares saudáveis e à prática regular de atividade física, garantindo a difusão de informação e a reflexão coletiva sobre os fatores individuais e coletivos que influenciam as práticas em saúde e nutrição na sociedade e estimulando o espírito crítico e o discernimento das pessoas.

O Brasil adota um modelo de reorientação da atenção primária à saúde, por meio da Estratégia Saúde da Família. É uma estratégia prioritária que visa atender a indivíduos e à família de forma integral e contínua, baseado na territorialização, desenvolvendo ações de promoção, proteção e recuperação da saúde. Tem como objetivo reorganizar a prática assistencial, centrada no hos- 
Ações realizadas no âmbito da atenção primária à saúde, que devem ser potencializadas nas três esferas de governo, para uma agenda única de nutrição.

\begin{tabular}{|c|c|c|}
\hline & $\begin{array}{l}\text { Prevenção e controle das carências nutricionais e dos distúrbios } \\
\text { associados à má-alimentação e práticas alimentares saudáveis }\end{array}$ & $\begin{array}{l}\text { Avaliação e monitoramento do estado nutricional } \\
\text { da população brasileira }\end{array}$ \\
\hline \multirow[t]{3}{*}{$\begin{array}{l}\text { Ações realizadas no } \\
\text { âmbito da atenção } \\
\text { primária à saúde }\end{array}$} & $\begin{array}{l}\text { 1. Suplementação medicamentosa de micronutrientes para grupos } \\
\text { mais vulneráveis como crianças, nutrizes e gestantes, universal ou } \\
\text { em regiões endêmicas, cuja carência seja considerada problema } \\
\text { de saúde pública (Portaria } n^{\circ} .730 \text {, } \\
\text { de } 13 \text { de maio de } 2005 \text { e Portaria } n^{\circ} .729 / G M \text {, de } \\
13 \text { de maio de } 2005 \text { ). }\end{array}$ & $\begin{array}{l}\text { 1. Sistema de Informação de Vigilância Alimentar e } \\
\text { Nutricional (SISVAN), no serviço de saúde (Sistema Único } \\
\text { de Saúde - SUS), para todas as fases do curso da vida } \\
\text { (Portaria } n^{\circ} .1 .156 \text {, de } 31 \text { de agosto de 1990). }\end{array}$ \\
\hline & $\begin{array}{l}\text { 2. Educação alimentar e nutricional no serviço de saúde, } \\
\text { considerando as diferentes fases do curso da vida e as condições } \\
\text { de saúde, a partir das diretrizes alimentares oficiais definidas nos } \\
\text { guias alimentares } 22,24 \text {. }\end{array}$ & $\begin{array}{l}\text { 2. Protocolo do Sistema de Informação de Vigilância } \\
\text { Alimentar e Nutricional (SISVAN): orientações para o } \\
\text { atendimento nutricional em serviços de saúde da atenção } \\
\text { básica. }\end{array}$ \\
\hline & $\begin{array}{l}\text { 3. Ações intersetoriais que possibilitem o acesso e a informação } \\
\text { sobre alimentação saudável, envolvendo parcerias especialmente } \\
\text { entre a educação, assistência social, desenvolvimento agrário e } \\
\text { saúde } 19,24 \text {. }\end{array}$ & $\begin{array}{l}\text { 3. Protocolos de atendimento para os problemas de } \\
\text { saúde mais prevalentes na região, com destaque para a } \\
\text { desnutrição, a obesidade e as outras doenças crônicas } \\
\text { não transmissíveis e outros distúrbios alimentares. } \\
\text { 4. Acompanhamento prioritário na atenção básica às } \\
\text { famílias beneficiárias do Programa Bolsa Família. }\end{array}$ \\
\hline
\end{tabular}

pital, com enfoque na família em seu ambiente físico e social. Também pode ser definida como um modelo de atenção que pressupõe o reconhecimento de saúde como um direito expresso na melhoria das condições de vida. No tocante à área da saúde, essa melhoria e sua qualificação deve ser traduzida em serviços mais resolutivos, integrais e especialmente humanizados 26 .

Recentemente, foi publicada a portaria que institui os Núcleos de Apoio à Saúde da Família (NASF) 27, que corresponde a uma iniciativa para apoiar as equipes mínimas da Estratégia Saúde da Família. Esses núcleos poderão ser compostos por médicos (ginecologistas, pediatras e psiquiatras), professores de educação física, nutricionistas, acupunturistas, homeopatas, farmacêuticos, assistentes sociais, fisioterapeutas, fonoaudiólogos, psicólogos e terapeutas ocupacionais. A instituição dos NASF permitirá a ampliação da abrangência e o escopo das ações bem como da resolubilidade.

Na lógica desse modelo de atenção, a agenda única da nutrição deve ser organizada pela caracterização clara do perfil epidemiológico da comunidade e dos espaços domiciliares com a identificação de riscos, problemas, prioridades, potencialidades e possibilidades de atuação e reconhecimento da situação de saúde, alimen- tação e nutrição das famílias, utilizando os diversos sistemas de informação da atenção básica, dentre os quais o SISVAN, que servirão de base para a realização do diagnóstico em nível local até a tomada de decisão em diferentes níveis de governo.

Após diagnóstico local, é possível realizar o planejamento com vistas à estruturação das ações de prevenção e controle das carências nutricionais e de promoção da alimentação saudável no serviço, de forma a contemplar as peculiaridades e as diversidades locais, assim como definir os mecanismos de apoio e os espaços intersetoriais, com destaque para o papel das escolas, dos equipamentos da rede de assistência social e do desenvolvimento agrário, como potenciais atores de atuação conjunta.

\section{Conclusão: desafios para o enfrentamento na agenda única}

O conjunto desses dados apresentados de diferentes grupos etários confirma que o Brasil convive concomitantemente com a desnutrição e com prevalências preocupantes de excesso de peso e obesidade, resultantes da má-alimentação. Essa situação não apenas afeta o sistema de 
Medidas coletivas desenvolvidas pelo setor saúde, que devem ser potencializadas nas três esferas de governo, para uma agenda única de nutrição.

Prevenção e controle das carências nutricionais e dos distúrbios associados à má-alimentação e práticas alimentares saudáveis

1. Fortificação universal de alimentos com micronutrientes (ferro, ácido fólico e iodo), cuja carência seja considerada problema de saúde pública (Resoluções RDC n. 130, de 26 de maio de 2003 e $\operatorname{RDC} n^{\circ} 344$, de 13 de dezembro de 2002).

2. Campanhas informativas sobre alimentação saudável, incentivando o aumento do consumo de frutas e hortaliças, no contexto de uma alimentação saudável 24 .

3. Medidas de apoio ao aleitamento materno e de promoção da alimentação complementar 24 .

4. Implementação das diretrizes da alimentação saudável no ambiente escolar: inserção do tema de forma transversal no currículo escolar; incentivo ao aumento do consumo de frutas e hortaliças; boas práticas de fabricação dos serviços de alimentação; restrição ao comércio de alimentos ricos em açúcar, sal, gorduras saturadas e trans; e monitoramento do estado nutricional dos escolares (Portaria Interministerial - Saúde e Educação n. 1.010, de 8 de maio de 2006) 24.

5. Aprimoramento da rotulagem nutricional obrigatória e, ainda, estratégias de educação que permitam o seu entendimento pela população (Resolução $\operatorname{RDC} n^{\circ}$. 359, de 26 de dezembro de 2003) 24 .

6. Criação de marcos legais para limitar o teor de açúcar, sal e gorduras saturadas e trans nos alimentos processados 24

7. Controle de todas as formas de publicidade e marketing de alimentos, voltadas para o público infantil 24
Avaliação e monitoramento do estado nutricional da população brasileira

1. Chamadas nutricionais: mobilização e sensibilização para a importância da vigilância alimentar e nutricional; avaliação com representatividade estadual do consumo alimentar e antropométrica de crianças.

2. Inquéritos populacionais periódicos de estado nutricional e de consumo alimentar: avaliação de consumo alimentar; avaliação antropométrica e; avaliação de carências nutricionais específicas saúde nacional, mas todo o contexto cultural, social e econômico do país, sendo reconhecidamente o maior impedimento à concretização do potencial humano.

A literatura aponta a necessidade de medidas de promoção da alimentação saudável como fundamental para a resolução da desnutrição e das deficiências de micronutrientes, ainda, da prevenção da obesidade e doenças crônicas não transmissíveis relacionadas que vão interromper a intergeração da desnutrição e pobreza 1. É im- portante que essas medidas estejam em consonância com as políticas nacionais que têm interface com os determinantes do estado nutricional de uma população e também com os programas de transferência de renda.

O desafio está posto: o fortalecimento da rede de nutrição no SUS institucionalmente, nas três esferas de governo, que possibilite a implementação de uma agenda única de nutrição, no foco da promoção da alimentação saudável, no enfoque do curso da vida? 


\section{Resumo}

O Brasil, assim como outros países em desenvolvimento, convive atualmente com a transição nutricional, determinada freqüentemente pela má-alimentação. Os inquéritos populacionais mostraram a tendência contínua de redução da desnutrição no país, associada ao aumento do excesso de peso em diferentes fases da vida. O monitoramento do perfil nutricional permite a geração de uma atitude de vigilância e o direcionamento das ações de promoção de saúde. A Estratégia Saúde da Família, em conjunto com diversos aspectos conjunturais do país, mostrou resultados concretos para a redução da desnutrição; contudo, a má-alimentação contribuiu de forma expressiva para o aumento da prevalência de obesidade e de outras doenças crônicas não transmissiveis, que, além de ter grande impacto na qualidade de vida do indivíduo, oneram significativamente o Sistema Único de Saúde. Para atuar nesse dilema da saúde pública contemporânea, é necessário priorizar uma agenda única de nutrição, no enfoque do curso da vida, quebrando um ciclo vicioso que se inicia ainda no período intra-uterino e se perpetua ao longo da vida.

Epidemiologia Nutricional; Transição Nutricional; Doenças Crônicas

\section{Referências}

1. Standing Committee on Nutrition. Diet-related chronic diseases and double burden of malnutrition in West Africa. London: United Nations System; 2006. (Standing Committee on Nutrition News, 33).

2. Batista Filho M, Rissin A. A transição nutricional no Brasil: tendências regionais e temporais. Cad Saúde Pública 2003; 19 Suppl 1:S181-91.

3. Bermudez OI, Tucker KL. Trends in dietary patterns of Latin American populations. Cad Saúde Pública 2003; 19 Suppl 1:S87-99.

4. World Health Organization. The World Health Report 2002: reducing risks, promoting healthy life. Geneva: World Health Organization; 2002.

5. Lessa I. Doenças crônicas não transmissíveis no Brasil: um desafio para a complexa tarefa da vigilância. Ciênc Saúde Coletiva 2004; 9:931-43.

6. Malta DC, Cezário AC, Moura L, Morais Neto OL, Silva Junior JB. A construção da vigilância e prevenção das doenças crônicas não transmissíveis no contexto do Sistema Único de Saúde. Epidemiol Serv Saúde 2006; 15:47-65.

\section{Colaboradores}

N. Toral descreveu o perfil nutricional da população brasileira no artigo. J. G. Coutinho e P. C. Gentil contribuíram com a discussão sobre a agenda única da nutrição no Brasil. Todas as autoras participaram das revisão final e formatação do trabalho.
7. Instituto Brasileiro de Geografia e Estatística. Pesquisa de Orçamentos Familiares 2002-2003: análise da disponibilidade domiciliar de alimentos e do estado nutricional no Brasil. Rio de Janeiro: Instituto Brasileiro de Geografia e Estatística; 2004.

8. Organización Mundial de la Salud. El estado físico: uso e interpretación de la antropometria. Informe de un Comité de Expertos de la OMS. Geneva: World Health Organization; 1995. (Serie de Informes Técnicos, 854).

9. Instituto Brasileiro de Geografia e Estatística. Pesquisa de Orçamentos Familiares 2002-2003: antropometria e análise do estado nutricional de crianças e adolescentes no Brasil. Rio de Janeiro: Instituto Brasileiro de Geografia e Estatística; 2006.

10. Departamento de Atenção Básica, Secretaria de Atenção à Saúde, Ministério da Saúde. Avaliação normativa do Programa Saúde da Família no Brasil: monitoramento da implantação e funcionamento das equipes de saúde da família - 20012002. Brasília: Ministério da Saúde; 2004. 
11. Macinko J, Guanais FC, Souza MFM. Evaluation of the impact of the Family Health Program on infant mortality in Brazil, 1990-2002. J Epidemiol Community Health 2006; 60:13-9.

12. de Onis M, Frongillo EA, Blössner N. Is malnutrition declining? An analysis of changes in levels of child malnutrition since 1980. Bull World Health Organ 2000; 78:1222-33.

13. Instituto de Pesquisa Econômica Aplicada. Objetivos de desenvolvimento do milênio: relatório nacional de acompanhamento. Brasília: Instituto de Pesquisa Econômica Aplicada; 2007.

14. Instituto Brasileiro de Geografia e Estatística. Pesquisa Nacional por Amostra de Domicílios 2003. Rio de Janeiro: Instituto Brasileiro de Geografia e Estatística; 2004.

15. Sociedade Civil Bem-Estar Familiar no Brasil. Pesquisa Nacional sobre Demografia e Saúde, 1996. $2^{\text {a }}$ Ed. Rio de Janeiro: Sociedade Civil Bem-Estar Familiar no Brasil; 1999.

16. Guigliani ERJ. Baixa estatura: um mal da sociedade brasileira. J Pediatr (Rio J) 1994; 70:261-2.

17. Laurentino GEC, Arruda IKG, Arruda BKG. Nanismo nutricional em escolares no Brasil. Rev Bras Saúde Matern Infant 2003; 3:377-85.

18. Monteiro CA, Conde WL, Konno SC. Análise do inquérito Chamada Nutricional 2005. In: Secretaria de Avaliação e Gestão da Informação, Ministério do Desenvolvimento Social e Combate à Fome, organizador. Chamada Nutricional: um estudo sobre a situação nutricional das crianças no semi-árido brasileiro. Brasília: Secretaria de Avaliação e Gestão da Informação, Ministério do Desenvolvimento Social e Combate à Fome; 2006. (Cadernos de Estudos: Desenvolvimento Social em Debate, 4).

19. Coordenação-Geral da Política de Alimentação e Nutrição, Departamento de Atenção Básica, Secretaria de Atenção à Saúde, Ministério da Saúde. Guia alimentar para a população brasileira: promovendo a alimentação saudável. Brasília: Ministério da Saúde; 2006.
20. Barker DJ, Eriksson JG, Forsen T, Osmond C. Fetal origins of adult disease: strength of effects and biological basis. Int J Epidemiol 2002; 31:1235-9.

21. Darnton-Hill I, Nishida C, James WP. A life course approach to diet, nutrition and the prevention of chronic diseases. Public Health Nutr 2004; 7(1A):101-21.

22. Departamento de Atenção Básica, Secretaria de Atenção à Saúde, Ministério da Saúde. Política nacional de alimentação e nutrição. 2a Ed. Brasília: Ministério da Saúde; 2005.

23. Brasil. Lei no ${ }^{\circ}$ 11.346, de 15 de setembro de 2006. Cria o Sistema Nacional de Segurança Alimentar e Nutricional - SISAN com vistas em assegurar o direito humano à alimentação adequada e dá outras providências. Diário Oficial da União 2006; 18 set.

24. World Health Organization. Integrated prevention of noncommunicable diseases: Global Strategy on Diet, Physical Activity and Health. http://www. who.int/dietphysicalactivity/strategy/eb11344/ en/index.html (acessado em 30/Nov/2007).

25. Uauy R, Monteiro C. The challenge of improving food and nutrition in Latin America. Food Nutr Bull 2004; 25:175-82.

26. Conselho Nacional de Secretários de Saúde. Atenção primária e promoção da saúde. Brasília: Conselho Nacional de Secretários de Saúde; 2007. (Coleção Pró-Gestores - Para Entender a Gestão do SUS).

27. Brasil. Portaria $\mathrm{n}^{\mathrm{o}}$. 154, de 24 de janeiro de 2008. Cria os Núcleos de Apoio à Saúde da Família - NASF. Brasília: Diário Oficial da União 2008; 25 jan.

Recebido em 04/Jan/2008

Versão final reapresentada em 30/Jun/2008

Aprovado em 03/Jul/2008 